\section{OPEN ACCESS}

Edited by:

loannis Zabetakis,

University of Limerick, Ireland

Reviewed by:

Pan Liu,

Northwestern University, United States Ivan Milos Stankovic,

University of Belgrade, Serbia

*Correspondence: Ricardo Wesley Alberca

ricardowesley@gmail.com

Specialty section:

This article was submitted to

Nutritional Immunology,

a section of the journal

Frontiers in Nutrition

Received: 31 March 2021

Accepted: 29 April 2021

Published: 02 June 2021

Citation:

Alberca RW, Rigato PO, Ramos YÁL,

Teixeira FME, Branco ACC,

Fernandes IG, Pietrobon $A J$

Duarte AJdS, Aoki V, Orfali RL and

Sato MN (2021) Clinical

Characteristics and Survival Analysis

in Frequent Alcohol Consumers With

COVID-19. Front. Nutr. 8:689296.

doi: 10.3389/fnut.2021.689296

\title{
Clinical Characteristics and Survival Analysis in Frequent Alcohol Consumers With COVID-19
}

\begin{abstract}
Ricardo Wesley Alberca ${ }^{1 *}$, Paula Ordonhez Rigato ${ }^{2}$, Yasmim Álefe Leuzzi Ramos ${ }^{1}$, Franciane Mouradian Emidio Teixeira ${ }^{1,3}$, Anna Cláudia Calvielli Branco ${ }^{1,3}$, lara Grigoletto Fernandes ${ }^{1}$, Anna Julia Pietrobon ${ }^{1,3}$, Alberto Jose da Silva Duarte ${ }^{1}$, Valeria Aoki ${ }^{1}$, Raquel Leão Orfali ${ }^{1}$ and Maria Notomi Sato ${ }^{1}$
\end{abstract}

\begin{abstract}
1 Laboratorio de Dermatologia e Imunodeficiencias (LIM-56), Departamento de Dermatologia, Faculdade de Medicina FMUSP, Instituto de Medicina Tropica, Universidade de São Paulo, São Paulo, Brazil, ${ }^{2}$ Technical Division of Medical Biology, Immunology Center, Adolfo Lutz Institute, São Paulo, Brazil, ${ }^{3}$ Department of Immunology, Institute of Biomedical Sciences, University of São Paulo, São Paulo, Brazil
\end{abstract}

Severe acute respiratory syndrome coronavirus-2 (SARS-CoV-2) infection can generate a systemic disease named coronavirus disease-2019 (COVID-19). Currently, the COVID-19 pandemic has killed millions worldwide, presenting huge health and economic challenges worldwide. Several risk factors, such as age, co-infections, metabolic syndrome, and smoking have been associated with poor disease progression and outcomes. Alcohol drinking is a common social practice among adults, but frequent and/or excessive consumption can mitigate the anti-viral and anti-bacterial immune responses. Therefore, we investigated if patients with self-reported daily alcohol consumption (DAC) presented alteration in the immune response to SARS-CoV-2. We investigated 122 patients with COVID-19 (101 male and 46 females), in which 23 were patients with DAC (18 men and 5 women) and 99 were non-DAC patients (58 men and 41 women), without other infections, neoplasia, or immunodeficiencies. Although with no difference in age, patients with DAC presented an increase in severity-associated COVID-19 markers such as C-reactive protein (CRP), neutrophil count, and neutrophil-to-lymphocyte ratio. In addition, patients with DAC presented a reduction in the lymphocytes and monocytes counts. Importantly, the DAC group presented an increase in death rate in comparison with the non-DAC group. Our results demonstrated that, in our cohort, DAC enhanced COVID-19-associated inflammation, and increased the number of deaths due to COVID-19.

Keywords: COVID-19, SARS-CoV-2, alcohol, consumption, inflammation

\section{INTRODUCTION}

Severe acute respiratory syndrome coronavirus-2 (SARS-CoV-2) infection can generate a severe systemic and multi-organ disease named coronavirus disease-2019 (COVID-19). SARS-CoV-2 has infected and killed millions worldwide (1). Several risk factors, such as co-infections (2), old age, chronic respiratory diseases, and comorbidities (3) have been associated with poor outcomes of COVID-19. 
During the pandemic, reports have highlighted the increase in alcohol consumption and the increased risk to trigger or exacerbate depressive and anxious episodes (4). Excessive consumption of alcohol can impair the immune response (5) present a deleterious effect in chronic viral infection $(6,7)$ and increase the severity and recovery time for respiratory infections (8-11).

The use of substances such as tobacco smoking is an established risk factor for severe COVID-19 (12), but few reports have identified people with alcohol use disorder or daily alcohol consumption (DAC) in their cohorts, with reports identifying no influence on neither drinking alcohol on severity nor death rates in patients with COVID-19 $(13,14)$. Therefore, we aimed to perform an investigation in our cohort to assess if DAC could influence the disease course and outcome of COVID-19.

\section{METHODS}

Patients were recruited fromat a special ward in the university hospital (Hospital das Clinicas da Universidade de São Paulo HCFMUSP) for moderate and severe patients with COVID-19. Inclusion criteria consisted of a positive diagnosise for COVID19 by the detection of SARS-CoV-2 RNA in nasopharyngeal swab by reverse-transcriptase PCR (RT-PCR). DAC was determined by self-report, some patients were clinically diagnosed with alcoholdependency prior to COVID-19, and some reported necessity for DAC of over two drinks.

Patients with and without DAC with type 2 Diabetes Mellitus (DM) and/or systemic arterial hypertension (SAH) were also included. The exclusion criteria were the presence of co-infections, renal or heart diseases, immunodeficiencies, or neoplasia. This investigation was approved by the Ethics Committee of HCFMUSP with approval for the usage of digital data from patients (no. 30800520.7.0000.0068-2020) and was performed in accordance with the 2013 revision of the Declaration of Helsinki. On the first day after hospitalization, EDTA plasma samples were obtained from a single venipuncture, and laboratory analysis was performed at the Central Laboratory of HCFMUSP (Divisão de Laboratório Central-HCFMUSP). Statistical analysis for age and laboratory data was performed using the Mann-Whitney test for comparisons between groups. Survival curve statistical analysis was performed using the Logrank (Mantel-Cox) test and the Gehan-Breslow-Wilcoxon test with GraphPad Prism 8 software (GraphPad Software, Inc., San Diego, CA).

Were included in this investigation 23 individuals with DAC (18 men and 5 women) and 99 patients with non-DAC (58 men and 41 women) that were hospitalized between June 2020 and 1 December 2020.

\section{RESULTS}

From the 23 DAC patients with COVID-19, 11 had moderate level COVID-19 and were admitted into the general ward (GW) $( \pm 48 \%)$ and 12 were admitted into the intensive care unit (ICU) $( \pm 52 \%)$ and needed assisted mechanical ventilation. It is important to highlight that among the 23 DAC patients with
COVID-19, one patient has DM $( \pm 4 \%)$, six patients have SAH $( \pm 26 \%)$, and five patients have both DM and SAH $( \pm 21 \%)$.

From the total 99 non-DAC patients with COVID-19, 52 were admitted into the GW $( \pm 52 \%)$ and 47 were admitted into the ICU $( \pm 47 \%)$. Among the 99 patients with COVID-19: 4 patients have DM $( \pm 4 \%), 23$ patients have SAH $( \pm 23 \%)$, and 24 patients have both DM and SAH $( \pm 24 \%)$.

The Patients with non-DAC and DAC did not present any age difference or significant statistical differences in the levels of alanine aminotransferase, aspartate aminotransferase, alkaline phosphatase, total bilirubin, direct bilirubin, indirect bilirubin, glutamyl transferase gamma, creatinine, lactate dehydrogenase, total protein and fractions, albumin, globulin, urea, and Ddimer, prothrombin time (Table 1). Nevertheless, both nonDAC and DAC presented alterations in alanine aminotransferase, aspartate aminotransferase, direct bilirubin, glutamyl transferase gamma, creatinine, lactate dehydrogenase, C-reactive protein (CRP), urea, D-dimer, and the prothrombin time were outside the considered normal range (Table 1).

We observed an increase in CRP and a reduction in platelets and activated partial thromboplastin time in patients with DAC in comparison to those with non-DAC (Table 1). This indicates an overall increase in COVID-19 biomarkers, such as urea, Ddimer, and creatinine levels in both groups, but a further increase in CRP levels showed a decrease in platelet counts, which was previously associated with COVID-19 severity $(15,16)$. The CRP level, especially at early hospitalization, has been associated with the extension of COVID-19-induced lung injury and disease progression, and death $(16,17)$. Models for predicting the disease outcome of COVID-19 are based on inflammatory markers on the first day of hospitalization (18).

Interestingly, although the total leukocyte count was similar among groups (Figure 1A), DAC groups presented an increase in the neutrophil and neutrophil to lymphocyte ratio (Figures 1B,D). Neutrophil count and the neutrophil-to lymphocyte-ratio are also established hallmarks of COVID-19 and are associated with disease severity (19, 20). An increase in neutrophils, as well as neutrophil migration to the lungs, is associated with lung inflammation and immunothrombosis in patients with COVID-19 (21). The neutrophil- to lymphocyteratio is a widely used maker for COVID-19 prognoses $(19,20,22,23)$, but the increase in the DAC group was due to both an increase in the neutrophil count (Figure 1B) and a reduction in the lymphocytes count in relation to the non-DAC group (Figure $1 \mathrm{C}$ ).

Lymphopenia, the reduction in the number of lymphocytes, is common in patients with COVID-19, with a significant reduction in T helper cells, $\mathrm{T}$ cytotoxic cells, Natural Killer, and B cells, impacting the anti-SARS-CoV-2 and other co-infections (2, 24, 25). In fact, lymphopenia is also associated with the severity and recovery from COVID-19 (26).

In our cohort, the number of monocytes in patients with DAC was reduced in comparison with patients with nonDAC (Figure 1E). Currently, reports have identified phenotypical alteration on monocytes of patients with COVID-19 (27), with a reduction in the antigen-presenting potential and an increase in the pro-inflammatory markers (28). Nevertheless, the reduction in monocytes of patients with DAC may also indicate 
TABLE 1 | Patients' clinical characteristics and laboratory data.

\begin{tabular}{|c|c|c|c|c|c|c|}
\hline & \multicolumn{2}{|c|}{ Non-DAC ( $N=99)$} & \multicolumn{2}{|c|}{$\mathrm{DAC}(N=23)$} & \multirow[b]{2}{*}{ Reference numbers } & \multirow[b]{2}{*}{$p$-value } \\
\hline & Mean & SEM & Mean & SEM & & \\
\hline Male/Female & $58 / 41$ & $18 / 5$ & & & & \\
\hline Age (years) & 55.08 & 1.335 & 56.38 & 3.135 & - & 0.2963 \\
\hline \multicolumn{7}{|l|}{ Laboratory data } \\
\hline Alanine aminotransferase (U/L) & 48.43 & 5.797 & 48.13 & 7.436 & $<41$ & 0.9238 \\
\hline Aspartate aminotransferase (U/L) & 43.44 & 4.149 & 51.42 & 9.261 & $<37$ & 0.7681 \\
\hline Alkaline phosphatase (U/L) & 115 & 18.89 & 107.2 & 15.78 & $40-129$ & 0.7865 \\
\hline Total bllirubin (mg/dL) & 0.4538 & 0.06193 & 0.4533 & 0.0485 & $0.2-1.0$ & 0.3681 \\
\hline Direct bilirubin (mg/dL) & 0.3578 & 0.0604 & 0.3183 & 0.03358 & $<0.3$ & 0.6342 \\
\hline Indirect bilirubin (mg/dL) & 0.09385 & 0.01291 & 0.03358 & 0.02393 & $0.1-0.6$ & 0.2229 \\
\hline Glutamyl transferase gamma (U/L) & 164.9 & 30.59 & 156.6 & 19.33 & $8-61$ & 0.4998 \\
\hline Creatinine (mg/dL) & 2.12 & 0.2077 & 2.042 & 0.3794 & $0.7-1.2$ & 0.9659 \\
\hline Lactate dehydrogenase (U/L) & 457.9 & 33.48 & 451.2 & 55.76 & $135-225$ & 0.4321 \\
\hline C-reactive protein (mg/L) & $77.54^{\star \star}$ & 9.205 & $154.2^{\star \star}$ & 27.97 & $<5.0$ & 0.0089 \\
\hline Total protein and fractions (g/dL) & 5.794 & 0.2328 & 6.37 & 0.2574 & $6.6-8.7$ & 0.1698 \\
\hline Albumin (g/dL) & 2.919 & 0.1046 & 3.255 & 0.1895 & $3.4-4.8$ & 0.2643 \\
\hline Globulin (g/dL) & 2.875 & 0.2065 & 3.085 & 0.1464 & $1.7-3.5$ & 0.3153 \\
\hline Urea (mg/dL) & 75.13 & 5.447 & 85.17 & 12.7 & $10-50$ & 0.5556 \\
\hline D-dimer (ng/mL) & 1963 & 252.2 & 1497 & 175.5 & $<500$ & 0.4949 \\
\hline Platelets $\left(\times 10^{3} / \mathrm{mm}^{3}\right)$ & $277.7^{*}$ & 15.51 & $206.6^{*}$ & 21.89 & $150-400$ & 0.0474 \\
\hline Prothrombin time (seconds) & 13.99 & 0.3263 & 13.08 & 0.3433 & $9.4-12.5$ & 0.1133 \\
\hline Activated partial thromboplastin time (seconds) & $38.87^{\star}$ & 2.408 & $28.84^{*}$ & 1.034 & $25.1-36.5$ & 0.0169 \\
\hline
\end{tabular}

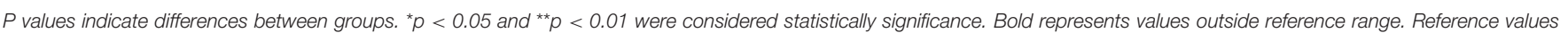
from Divisão de Laboratorio Central do HC/FMUSP.

a deficient immune response to SARS-CoV-2, as monocytes have a fundamental role in the immune response to pathogenic microorganisms (29).

We identified a reduction in both eosinophils and basophils in the DAC group in comparison with the non-DAC (Figures 1F,G). Low eosinophil and basophil counts have been associated with the worst anti-SARS-CoV-2 response (30).

We failed to identify differences in hospitalization time of the DAC group, which was $29.6 \pm 6.1$ days and the non-DAC group, which was $29.8 \pm 3$ days. It is noteworthy that a significant increase in death rate in the DAC group in comparison with the non-DAC group (Figure 2) $[p=0.0417$ using the Log-rank (Mantel-Cox) test and $p=0.0209$ using the Gehan-BreslowWilcoxon test]. During hospitalization, 8 patients from the DAC group and 14 patients from the non-DAC group passed away due to COVID-19 besides the efforts of the medical staff.

\section{DISCUSSION}

Excessive alcohol consumption can increase the severity and recovery time for infections (8). Several reports have highlighted the negative impact of excessive alcohol consumption on the bacterial respiratory infection (31) and viral respiratory infections such as respiratory syncytial virus (9) and influenza (10).

The increased risk for respiratory viral infections is due to the immune modulation caused by alcohol consumption $(8,10)$.
A previous report identified an increase in neutrophils and tumor necrosis factor- $\alpha$ (TNF) and monocyte chemoattractant protein-1 in the lungs of mice after alcohol consumption (32). Importantly, neutrophil count and TNF levels are associated with severe COVID-19 infection (33). In our cohort, patients with DAC presented a significant increase in the neutrophil and neutrophil to lymphocyte ratio, indicating a possibility that the frequent or excessive alcohol could further increase the severity of the infection caused by SARS-CoV-2.

Another hallmark alteration in chronic alcohol consumption is the reduction in the number and activity of NK cells, T cells, and $\mathrm{B}$ cells, decreasing the overall immune response and antibody production (34). This study verified that patients with DAC suffered from a significant reduction in the lymphocyte count in comparison to non-DAC and, that alcohol consumption was further detrimental to the lymphopenia suffered by patients with COVID-19 (22).

Short-term alcohol consumption can also impair the monocyte immune response, with an increase in the production of inflammatory cytokines but a decrease in the activation of the inflammatory complex by microbial components (35). Monocytes isolated from individuals after alcohol consumption present an increase in the activation of the inflammatory complex (inflammasome) and production of pro-inflammatory cytokine, IL-1 $\beta$ (36).

Importantly, patients with COVID-19 also present phenotypical alteration on monocytes, increased proinflammatory response, and reduced antigen presentation 


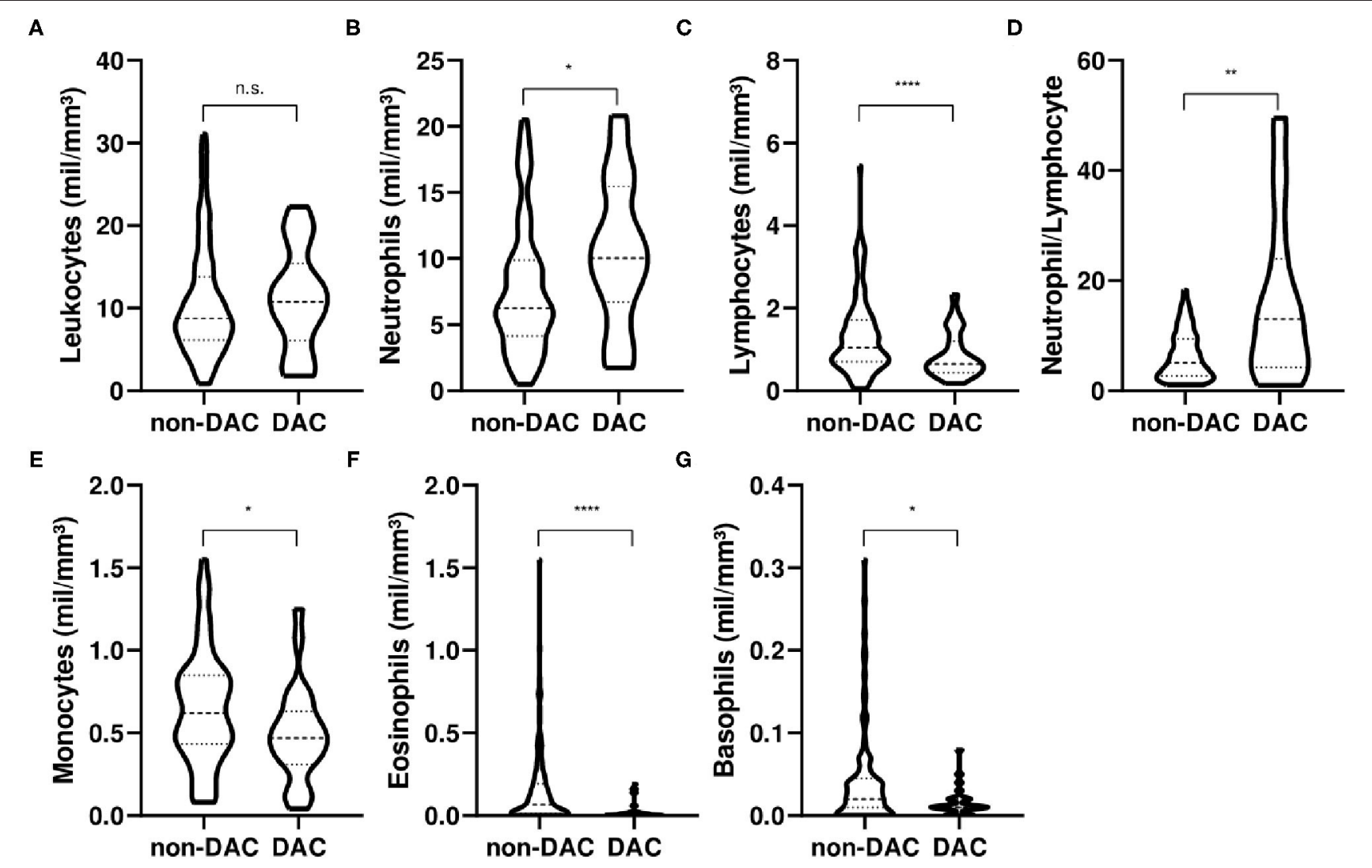

FIGURE 1 | Clinical features of patients with COVID-19. (A) Leukocyte counts, (B) Neutrophil counts, (C) Lymphocyte counts, (D) Neutrophil-to-lymphocyte ratio, (E) Monocyte counts, (F) Eosinophil counts, and (G) Basophil counts from the first day of hospitalization due to the SARS-CoV-2 infection. DAC, patients with self-reported daily alcohol consumption (DAC) and COVID-19; Non-DAC, patients without DAC and COVID-19. ${ }^{\star} p<0.05,{ }^{* *} p<0.01,{ }^{\star \star \star *} p<0.0001$, n.S., non-statistically significant difference. Mann-Whitney test used for comparisons. Data collected between June 1, 2020 and December 1, 2020.

potential $(27,28)$. Although the role of monocytes' on COVID19 is still under investigation, alcohol consumption may exacerbate the dysregulated profile of monocyte on COVID-19.

The long-term consumption of alcoholic beverages has been associated with longer hospitalization and intensive care usage upon respiratory infections (8). In our cohort, we did not identify any differences in hospitalization time between DAC and nonDAC groups.

Alcohol consumption is also associated with a reduction of anti-inflammatory molecules, such as IL-10 (37), which may further aggravate the COVID-19-associated lung inflammation and established dysregulation in the anti-inflammatory immune response (24). Besides, alcohol consumption modulates the gut microbiome (38), and curbs the viral immune response (39), which may expose the individual to SARS-CoV-2 gastrointestinal infection $(40,41)$, leading to, increased gastrointestinal dysbiosis (42) and more severe respiratory complication due to COVID19 (43).

Excessive alcohol consumption also impacts the absorption of nutrients, with a reduction in essential minerals and vitamins (44). Chronic alcohol consumption can impair vitamin A (45) and vitamin D (46) levels. Vitamin A deficiency reduces B-and
T-cell immune response to influenza in mice $(47,48)$, and could further enhance the COVID-19-mediated immune dysregulation (24). Vitamin D deficiency has been associated with an increase in respiratory distress syndrome (49), COVID-19 severity (50), and deaths (51).

Although to our knowledge, no investigation on the angiotensin-converting enzyme (ACE2) expression in the lungs has been performed following alcohol consumption, investigations have identified that chronic alcohol consumption can increase the expression of ACE in the blood (52) and angiotensin II type 2 (AT2) receptor in the lungs (53). This leads to the hypothesis that alcohol consumption might modulate the ACE2 receptor, the entry receptor of SARS-CoV-2, in a similar way to other diseases, either increasing or reducing COVID-19 risk (54-56).

It is noteworthy that we identified an increase in mortality in the DAC group in comparison to the non-DAC group due to COVID-19 respiratory complications, which could indicate that frequent alcohol consumption may increase the death risk by COVID-19.

This study possesses two limitations: (1) the quantity of alcohol ingested by members of the DAC group may vary and 


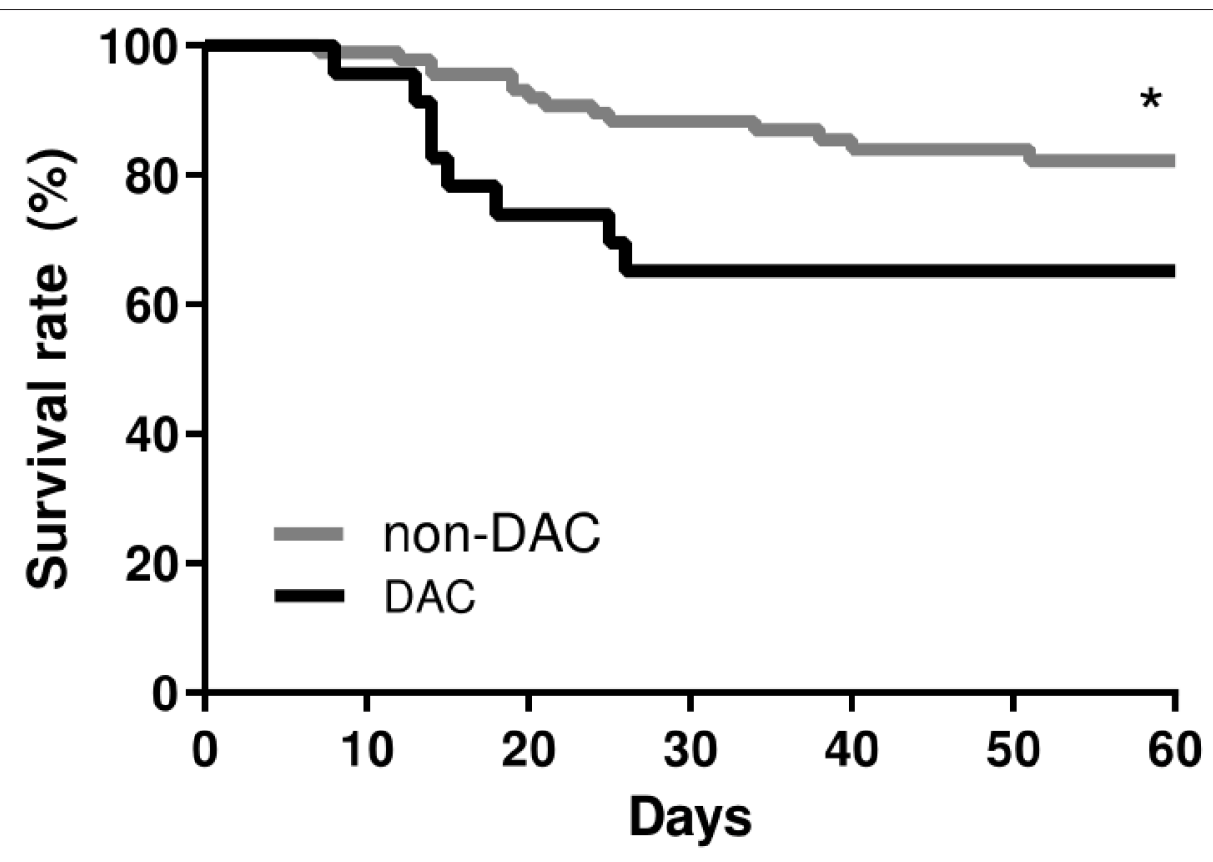

FIGURE 2 | Survival curve of patients with COVID-19. DAC, patients with self-reported DAC and COVID-19; Non-DAC, patients without DAC and COVID-19. * $P$, 0.05 difference. Comparison using the Log-rank (Mantel-Cox) test and the Gehan-Breslow-Wilcoxon test. Data collected between June 1, 2020 and December 1 , 2020.

could be an important variant; (2) it was impossible to isolate DAC from other comorbidities (SAH and/or DM) due to the number of individuals with DAC. Therefore, it is possible that the synergic effect of alcohol consumption with comorbidities influenced the COVID-19 outcome.

\section{CONCLUSION}

In summary, in our cohort, frequent alcohol consumption alters the COVID-19 clinical characteristics, further increasing proinflammatory markers and increasing the mortality risk due to COVID-19-associated complications. Due to the limited number of patients, further investigations should explore the possible synergic effect of alcohol consumption with other comorbidities on COVID-19 severity and mortality.

\section{DATA AVAILABILITY STATEMENT}

The original contributions presented in the study are included in the article/supplementary material, further inquiries can be directed to the corresponding author/s.

\section{REFERENCES}

1. WHO Coronavirus Disease (COVID-19). Dashboard. WHO Coronavirus Disease (COVID-19) Dashboard. Available online at: https://covid19.who.int/ (accessed September 21, 2020).

2. Alberca RW, Yendo TM, Leuzzi Ramos YÁ, Fernandes IG, Oliveira LM, Teixeira FME, et al. Case report: COVID-19

\section{ETHICS STATEMENT}

The studies involving human participants were reviewed and approved by Ethics Committee of Hospital das Clínicas da Faculdade de Medicina da Universidade de São Paulo-HCFMUSP with approval for the usage of digital data from patients (no. 30800520.7.0000.0068-2020). The patients/participants provided their written informed consent to participate in this study.

\section{AUTHOR CONTRIBUTIONS}

All authors contributed to data collection, analysis, write and review of the article, and approved the submitted version.

\section{FUNDING}

This study was supported by the Fundação de Amparo à Pesquisa do Estado de São Paulo (FAPESP). Grant Numbers: 19/026797, 17/18199-9. Coordenação de Aperfeiçoamento de Pessoal de Nível Superior-CAPES: 88887.503842/2020-00. 
4. Garcia LP, Sanchez ZM. Alcohol consumption during the COVID-19 pandemic: a necessary reflection for confronting the situation. Cad Saude Publ. (2020) 36:e00124520. doi: 10.1590/0102-311X00124520

5. Sarkar D, Jung MK, Wang HJ. Alcohol and the immune system. Alcohol Res Curr Rev. (2015) 298:543-4. doi: 10.1136/bmj.298.6673.543

6. Monnig MA. Immune activation and neuroinflammation in alcohol use and HIV infection: evidence for shared mechanisms. Am J Drug Alcohol Abuse. (2017) 43:7-23. doi: 10.1080/00952990.2016.1211667

7. Khan KN, Yatsuhashi H. Effect of alcohol consumption on the progression of hepatitis $\mathrm{C}$ virus infection and risk of hepatocellular carcinoma in Japanese patients. Alcohol Alcohol. (2000) 35:286-95. doi: 10.1093/alcalc/35.3.286

8. Saitz R, Ghali WA, Moskowitz MA. The impact of alcohol-related diagnoses on pneumonia outcomes. Arch Intern Med. (1997) 157:144652. doi: 10.1001/archinte.157.13.1446

9. Wyatt TA, Bailey KL, Simet SM, Warren KJ, Sweeter JM, DeVasure JM, et al. Alcohol potentiates RSV-mediated injury to ciliated airway epithelium. Alcohol. (2019) 80:17-24. doi: 10.1016/j.alcohol.2018.07.010

10. Greenbaum A, Chaves SS, Perez A, Aragon D, Bandyopadhyay A, Bennett $\mathrm{N}$, et al. Heavy alcohol use as a risk factor for severe outcomes among adults hospitalized with laboratory-confirmed influenza, 2005-2012. Infection. (2014) 42:165-70. doi: 10.1007/s15010-013-0534-8

11. Happel KI, Nelson S. Alcohol, immunosuppression, and the lung. Proc Am Thorac Soc. (2005) 2:428-32. doi: 10.1513/pats.200507-065JS

12. Alberca RW, Lima JC, de Oliveira EA, Gozzi-Silva SC, Ramos YÁL, de Andrade MMS, et al. COVID-19 disease course in former smokers, smokers and COPD patients. Front Physiol. (2021) 11:637627. doi: 10.3389/fphys.2020.637627

13. Zhong R, Chen L, Zhang Q, Li B, Qiu Y, Wang W, et al. Which factors, smoking, drinking alcohol, betel quid chewing, or underlying diseases, are more likely to influence the severity of COVID-19? Front Physiol. (2020) 11:623498. doi: 10.3389/FPHYS.2020.623498

14. Dai M, Tao L, Chen Z, Tian Z, Guo X, Allen-Gipson DS, et al. Influence of cigarettes and alcohol on the severity and death of COVID-19: a multicenter retrospective study in Wuhan, China. Front Physiol. (2020) 11:588553. doi: 10.3389/FPHYS.2020.588553

15. Bongiovanni D, Klug M, Lazareva O, Weidlich S, Biasi M, Ursu S, et al. SARSCoV-2 infection is associated with a pro-thrombotic platelet phenotype. Cell Death Dis. (2021) 12:1-10. doi: 10.1038/s41419-020-03333-9

16. Wang L. C-reactive protein levels in the early stage of COVID-19. Med Mal Infect. (2020) 50:332-4. doi: 10.1016/j.medmal.2020.03.007

17. Sharifpour M, Rangaraju S, Liu M, Alabyad D, Nahab FB, CreelBulos CM, et al. C-Reactive protein as a prognostic indicator in hospitalized patients with COVID-19. PLoS ONE. (2020) 15:e0242400. doi: 10.1371/journal.pone.0242400

18. Yildiz H, Yombi JC, Castanares-Zapatero D. Validation of a risk score to predict patients at risk of critical illness with COVID-19. Infect Dis. (2020) 53:137-8. doi: 10.1080/23744235.2020.1823469

19. Liu J, Liu Y, Xiang P, Pu L, Xiong H, Li C, et al. Neutrophil-to-lymphocyte ratio predicts critical illness patients with 2019 coronavirus disease in the early stage. J Transl Med. (2020) 18:206. doi: 10.1186/s12967-020-02374-0

20. Lian J, Jin C, Hao S, Zhang X, Yang M, Jin X, et al. High neutrophilto-lymphocyte ratio associated with progression to critical illness in older patients with COVID-19: a multicenter retrospective study. Aging. (2020) 12:13849-59. doi: 10.18632/aging.103582

21. Middleton EA, He X-Y, Denorme F, Campbell RA, Ng D, Salvatore SP, et al. Neutrophil extracellular traps (NETs) contribute to immunothrombosis in COVID-19 acute respiratory distress syndrome. Blood. (2020) 136:116979. doi: 10.1182/blood.2020007008

22. Alberca RW, de Andrade MMS, Castelo Branco ACC, Pietrobon AJ, Pereira NZ, Fernandes IG, et al. Frequencies of CD33+ CD11b+ HLA-DR- CD14CD66b + and CD33+ CD11b + HLA-DR- CD14+ CD66b- cells in peripheral blood as severity immune biomarkers in COVID-19. Front Med. (2020) 7:580677. doi: 10.3389/FMED.2020.580677

23. Zhang B, Zhou X, Zhu C, Song Y, Feng F, Qiu Y, et al. Immune phenotyping based on the neutrophil-to-lymphocyte ratio and IgG level predicts disease severity and outcome for patients with COVID-19. Front Mol Biosci. (2020) 7:157. doi: 10.3389/fmolb.2020.00157
24. Qin C, Zhou L, Hu Z, Zhang S, Yang S, Tao Y, et al. Dysregulation of immune response in patients with coronavirus 2019 (COVID-19) in Wuhan, China. Clin Infect Dis. (2020) 71:762-8. doi: 10.1093/cid/ciaa248

25. Langford BJ, So M, Raybardhan S, Leung V, Westwood D, MacFadden DR, et al. Bacterial co-infection and secondary infection in patients with COVID19: a living rapid review and meta-analysis. Clin Microbiol Infect. (2020) 26:1622-9. doi: 10.1016/j.cmi.2020.07.016

26. Liu J, Li H, Luo M, Liu J, Wu L, Lin $X$, et al. Lymphopenia predicted illness severity and recovery in patients with COVID19: A single-center, retrospective study. PLoS ONE. (2020) 15:e0241659. doi: 10.1371/journal.pone.0241659

27. Lombardi A, Trombetta E, Cattaneo A, Castelli V, Palomba E, Tirone M, et al. Early phases of COVID-19 are characterized by a reduction in lymphocyte populations and the presence of atypical monocytes. Front Immunol. (2020) 11:560330. doi: 10.3389/fimmu.2020.560330

28. Qin S, Jiang Y, Wei X, Liu X, Guan J, Chen Y, et al. Dynamic changes in monocytes subsets in COVID-19 patients. Hum Immunol. (2020) 82:1706. doi: 10.1016/j.humimm.2020.12.010

29. Shi C, Pamer EG. Monocyte recruitment during infection and inflammation. Nat Rev Immunol. (2011) 11:762-74. doi: 10.1038/nri3070

30. Rodriguez L, Pekkarinen PT, Lakshmikanth T, Tan Z, Consiglio CR, Pou C, et al. Systems-level immunomonitoring from acute to recovery phase of severe COVID-19. Cell Reports Med. (2020) 1:100078. doi: 10.1016/j.xcrm.2020.100078

31. De Roux A, Cavalcanti M, Marcos MA, Garcia E, Ewig S, Mensa J, et al. Impact of alcohol abuse in the etiology and severity of community-acquired pneumonia. Chest. (2006) 129:1219-25. doi: 10.1378/chest.129.5.1219

32. Jerrells TR, Pavlik JA, DeVasure J, Vidlak D, Costello A, Strachota JM, et al. Association of chronic alcohol consumption and increased susceptibility to and pathogenic effects of pulmonary infection with respiratory syncytial virus in mice. Alcohol. (2007) 41:357-69. doi: 10.1016/j.alcohol.2007.07.001

33. Li X, Xu S, Yu M, Wang K, Tao Y, Zhou Y, et al. Risk factors for severity and mortality in adult COVID-19 inpatients in Wuhan. J Allergy Clin Immunol. (2020) 146:110-8. doi: 10.1016/j.jaci.2020.04.006

34. Pasala S, Barr T, Messaoudi I. Impact of alcohol abuse on the adaptive immune system. Alcohol Res. (2015) 37:185-97.

35. Szabo G. Monocytes, alcohol use, and altered immunity. Alcohol Clin Exp Res. (1998) 22:216S-9S. doi: 10.1111/j.1530-0277.1998.tb04005.x

36. Lippai D, Bala S, Catalano D, Kodys K, Szabo G. Micro-RNA-155 deficiency prevents alcohol-induced serum endotoxin increase and small bowel inflammation in mice. Alcohol Clin Exp Res. (2014) 38:221724. doi: 10.1111 /acer. 12483

37. Marshall SA, McKnight KH, Blose AK, Lysle DT, Thiele TE. Modulation of binge-like ethanol consumption by IL-10 signaling in the basolateral amygdala. J Neuroimmune Pharmacol. (2017) 12:249-59. doi: 10.1007/s11481-016-9709-2

38. Litwinowicz K, Choroszy M, Waszczuk E. Changes in the composition of the human intestinal microbiome in alcohol use disorder: a systematic review. Am J Drug Alcohol Abuse. (2020) 46:4-12. doi: 10.1080/00952990.2019.1669629

39. Saltzman ET, Palacios T, Thomsen M, Vitetta L. Intestinal microbiome shifts, dysbiosis, inflammation, and non-alcoholic fatty liver disease. Front Microbiol. (2018) 30:61. doi: 10.3389/fmicb.2018.00061

40. Zuo T, Zhang F, Lui GCY, Yeoh YK, Li AYL, Zhan H, et al. Alterations in gut microbiota of patients with COVID-19 during time of hospitalization. Gastroenterology. (2020) 159:944-55.e8. doi: 10.1053/j.gastro.2020.05.048

41. Gupta S, Parker J, Smits S, Underwood J, Dolwani S. Persistent viral shedding of SARS-CoV-2 in faeces - a rapid review. Color Dis. (2020) 22:61120. doi: $10.1111 /$ codi. 15138

42. Alberca GGF, Solis-Castro RL, Solis-Castro ME, Alberca RW. Coronavirus disease-2019 and the intestinal tract: An overview. World J Gastroenterol. (2021) 27:1255-66. doi: 10.3748/wjg.v27.i13.1255

43. Anand S, Mande SS. Diet, microbiota and gut-lung connection. Front Microbiol. (2018) 19:2147. doi: 10.3389/fmicb.2018.02147

44. Gibson A, Woodside JV, Young IS, Sharpe PC, Mercer C, Patterson CC, et al. Alcohol increases homocysteine and reduces $\mathrm{B}$ vitamin concentration in healthy male volunteers - A randomized, crossover intervention study. QJM. (2008) 101:881-7. doi: 10.1093/qjmed/hcn112 
45. Clugston RD, Blaner WS. The adverse effects of alcohol on vitamin A metabolism. Nutrients. (2012) 4:356-71. doi: 10.3390/nu4050356

46. Lieber CS. Alcohol: its metabolism and interaction with nutrients. Annu Rev Nutr. (2000) 20:395-430. doi: 10.1146/annurev.nutr.20.1.395

47. Penkert RR, Smith AP, Hrincius ER, McCullers JA, Vogel P, Smith AM, et al. Effect of Vitamin A deficiency in dysregulating immune responses to influenza virus and increasing mortality rates after bacterial coinfections. J Infect Dis. (2020). doi: 10.1093/infdis/jiaa597. [Epub ahead of print].

48. Schmidt R, Luboeinski T, Markart P, Ruppert C, Daum C, Grimminger F, et al. Alveolar antioxidant status in patients with acute respiratory distress syndrome. Eur Respir J. (2004) 24:994-9. doi: 10.1183/09031936.04.001 20703

49. Dancer RCA, Parekh D, Lax S, D'Souza V, Zheng S, Bassford CR, et al. Vitamin D deficiency contributes directly to the acute respiratory distress syndrome (ARDS). Thorax. (2015) 70:617-24. doi: 10.1136/thoraxjnl-2014-2 06680

50. Ali N. Role of vitamin D in preventing of COVID-19 infection, progression and severity. I Infect Public Health. (2020) 13:137380. doi: 10.1016/j.jiph.2020.06.021

51. Radujkovic A, Hippchen T, Tiwari-Heckler S, Dreher S, Boxberger M, Merle U. Vitamin D deficiency and outcome of COVID-19 patients. Nutrients. (2020) 12:2757. doi: 10.3390/nu12092757

52. Okuno F, Arai M, Ishii H, Shigeta Y, Ebihara Y, Takagi S, et al. Mild but prolonged elevation of serum angiotensin converting enzyme (ACE) activity in alcoholics. Alcohol. (1986) 3:357-9. doi: 10.1016/0741-8329(86)9 0053-4
53. Bechara RI, Brown LAS, Eaton DC, Roman J, Guidot DM. Chronic ethanol ingestion increases expression of the angiotensin II Type 2 (AT2) receptor and enhances tumor necrosis factor-alpha- and angiotensin II-induced cytotoxicity via AT2 signaling in rat alveolar epithelial cells. Alcohol Clin Exp Res. (2003) 27:1006-14. doi: 10.1097/01.alc.0000071932.56932.53

54. Castelo Branco ACC, Sato MN, Alberca RW. The possible dual role of the ACE2 receptor in asthma and SARS-COV2 infection. Front Cell Infect Microbiol. (2020) 10:550571. doi: 10.3389/FCIMB.2020.550571

55. Jacobs M, van Eeckhoutte HP, Wijnant SRA, Janssens W, Brusselle GG, Joos $\mathrm{GF}$, et al. Increased expression of ACE2, the SARS-CoV-2 entry receptor, in alveolar and bronchial epithelium of smokers and COPD subjects. Eur Respir J. (2020) 56:2002378. doi: 10.1183/13993003.02378-2020

56. Alberca RW, Yendo T, Aoki V, Sato MN. Asthmatic patients and COVID-19: different disease course? Allergy. (2020) 76:963-5. doi: 10.1111/all.14601

Conflict of Interest: The authors declare that the research was conducted in the absence of any commercial or financial relationships that could be construed as a potential conflict of interest.

Copyright (C) 2021 Alberca, Rigato, Ramos, Teixeira, Branco, Fernandes, Pietrobon, Duarte, Aoki, Orfali and Sato. This is an open-access article distributed under the terms of the Creative Commons Attribution License (CC BY). The use, distribution or reproduction in other forums is permitted, provided the original author(s) and the copyright owner(s) are credited and that the original publication in this journal is cited, in accordance with accepted academic practice. No use, distribution or reproduction is permitted which does not comply with these terms. 